\title{
Filaggrin Gene Mutation c.3321delA Is Associated with Various Clinical Features of Atopic Dermatitis in the Chinese Han Population
}

\author{
Li Meng 1,2,39, Li Wang 1,2,39, Huayang Tang 1,2,39, Xianfa Tang 1,2,3, Xiaoyun Jiang ${ }^{1,2,3}$, Jinhua Zhao, 2,3, \\ Jing Gao ${ }^{1,2,3}$, Bing $\mathrm{Li}^{1,2,3}$, Xuhui $\mathrm{Fu}^{1,2,3}$, Yan Chen ${ }^{1,2,3}$, Weiyi Yao ${ }^{1,2,3}$, Wenying Zhan ${ }^{1,2,3}$, Bo Wu $\mathbf{W u}^{1,2,3}$, \\ Dawei Duan ${ }^{1,2,3}$, Changbing Shen ${ }^{1,2,3}$, Hui Cheng ${ }^{1,2,3}$, Xianbo Zuo ${ }^{1,2,3}$, Sen Yang ${ }^{1,2,3}$, Liangdan Sun ${ }^{1,2,3 *}$, \\ Xuejun Zhang ${ }^{1,2,3,4,5 *}$
}

1 Institute of Dermatology and Department of Dermatology, No.1 Hospital, Anhui Medical University, Hefei, Anhui, China, 2 Key Laboratory of Dermatology, Anhui Medical University, Ministry of Education, China, Hefei, Anhui, China, $\mathbf{3}$ State Key Laboratory Incubation Base of Dermatology, Anhui Medical University, Hefei, Anhui, China, 4 Department of Dermatology at No.2 Hospital, Anhui Medical University, Hefei, Anhui, China, 5 Department of Dermatology, Huashan Hospital of Fudan University, Shanghai, China

\begin{abstract}
Background: We confirmed that the filaggrin gene mutation c.3321 delA is associated with atopic dermatitis in our previous genome wide association study of the Chinese Han population. c.3321 delA is the most common filaggrin gene mutation in Chinese atopic dermatitis patients but is not present in European populations.

Objective: To investigate the genetic model for the c.3321delA mutation and to determine the correlation between c.3321 delA and atopic dermatitis clinical phenotypes in the Chinese Han population.

Method: The filaggrin gene mutation c.3321delA was sequenced in 1,080 atopic dermatitis patients and 908 controls from the Chinese population. The $\chi^{2}$ test, ANOVA,nonparametric tests and logistic regression were used to investigate the relationship between the c.3321delA genotype and atopic dermatitis clinical phenotypes in the Chinese Han population.

Results: Analyses of the genetic model revealed that the additive model best described the c.3321delA mutation $(P=3.09 \mathrm{E}-$ $11, \mathrm{OR}=3.43,95 \% \mathrm{Cl}=2.38-4.96)$. Stratified analyses showed that the c.3321delA allele frequency distribution is significantly associated with concomitant skin xerosis $(P=1.68 \mathrm{E}-03, \mathrm{OR}=2.13,95 \% \mathrm{Cl}=1.32-3.46)$, palmar hyperlinearity $(P=3.64 \mathrm{E}-17$, $\mathrm{OR}=4.0,95 \% \mathrm{Cl}=2.86-5.70)$, white dermatographism $(P=4.25 \mathrm{E}-03, \mathrm{OR}=1.82,95 \% \mathrm{Cl}=1.22-2.71)$, food intolerance $(P=1.51 \mathrm{E}-$ $03, \mathrm{OR}=1.76,95 \% \mathrm{Cl}=1.23-2.50)$ and disease severity $(P=9.67 \mathrm{E}-05)$.

Conclusion: Our study indicates that the filaggrin gene mutation c.3321delA is associated with clinical phenotypes of atopic dermatitis in the Chinese Han population, which might help us gain a better understanding on the pathogenesis of atopic dermatitis.
\end{abstract}

Citation: Meng L, Wang L, Tang H, Tang X, Jiang X, et al. (2014) Filaggrin Gene Mutation c.3321delA Is Associated with Various Clinical Features of Atopic Dermatitis in the Chinese Han Population. PLoS ONE 9(5): e98235. doi:10.1371/journal.pone.0098235

Editor: Yong-Gang Yao, Kunming Institute of Zoology, Chinese Academy of Sciences, China

Received January 7, 2014; Accepted April 30, 2014; Published May 23, 2014

Copyright: (c) 2014 Meng et al. This is an open-access article distributed under the terms of the Creative Commons Attribution License, which permits unrestricted use, distribution, and reproduction in any medium, provided the original author and source are credited.

Funding: This study was funded by General Program of National Natural Science Foundation of China $(31171224,31000528)$, Program for New Century Excellent Talents in University (NCET-11-0889), Science and Technological Foundation of Anhui Province for Outstanding Youth (1108085J10), Pre-project of State Key Basic Research Program 973 of China (No. 2012CB722404), and Hospital incubation program (3101005002354). The funders had no role in study design, data collection and analysis, decision to publish, or preparation of the manuscript.

Competing Interests: Xuejun Zhang is a PLOS ONE Editorial Board member. This does not alter the authors' adherence to PLOS ONE Editorial policies on sharing data and materials.

*E-mail: ayzxj@vip.sina.com (X. Zhang); ahmusld@163.com (LS)

9 These authors contributed equally to this work.

\section{Introduction}

Atopic dermatitis (AD) has long been recognized as a complex trait, wherein multiple genes and environmental stimuli contribute to disease manifestation. To date, 81 genes have been implicated in over 100 published reports on AD genetic association studies, and 46 of these genes have demonstrated at least one positive association with $\mathrm{AD}$. Of these genes, filaggrin gene $(F L G)$ is the most consistently replicated gene, appearing in 20 reports [1].
Filaggrin, also known as filament-aggregating protein, plays a major role in the epidermal barrier function. To date, approximately 60 loss-of-function FLG mutations have been identified in European and Asian populations [2]. All of the mutations that are predicted to cause loss of function, including nonsense mutations as well as out-of-frame insertions or deletions, are specific to certain ethnic groups, with distinct profiles observed in the European and Asian populations that have been well studied [3]. 
$\mathrm{AD}$, with a prevalence of 1.4 to $22.3 \%$ worldwide [4], has a complex clinical phenotype strongly associated with food allergies, asthma, and allergic rhinitis (AR) in a patient's life (i.e., the atopic march) [5]. Over the last decade, numerous association studies on FLG mutations and $\mathrm{AD}$-associated phenotypes have been conducted. The majority of the studies focused on combined FLG mutations, and only a few studies referred to single mutation. c.3321delA is an Asian-specific FLG mutation that has been described in Chinese, Japanese, Korean and Singaporean populations [6]. c.3321 delA is the most common FLG mutation in the Chinese population; however, it is not found in European populations. In our previous Genome Wide Association Study (GWAS) of AD, we identified the $F L G$ variant rs3126085 that correlates with c.3321delA. In this study, we investigated the genetic model for c.3321delA, the genotype-phenotype correlation between c.3321delA and AD in the Chinese population. This study employs the largest sample size of all of the genotypephenotype correlation studies of $\mathrm{AD}$ to date.

\section{Materials and Methods}

\section{Patients and controls}

A total of 1,080 AD patients and 908 controls were enrolled in this study (Table 1). All samples were from the Chinese Han population and were used in our previous GWAS. AD patients meeting the Hanifin-Rajka diagnostic criteria [7] were recruited from the No. 1 Hospital of Anhui Medical University and the Xinhua Hospital affiliated with the Shanghai Jiaotong University School of Medicine in China. Physician specialists collected clinical data from the affected individuals through a full clinical checkup. Additional demographic information was collected from the cases and controls through a structured questionnaire. The disease severity was evaluated using the objective SCORing Atopic Dermatitis (SCORAD) index [8], which categorizes patients as mild ( $\leq 15$ points), moderate (15-40 points) and severe $(>40$ points). Patients were considered to have food intolerance either evaluated by allergen test of venous blood samples or patients'selfreports. All controls were clinically assessed to be without $\mathrm{AD}$ or other atopic diseases, a family history of atopic diseases (including first-, second- and third-degree relatives) or ichthyosis vulgaris (IV). All participants provided written informed consent. The study was approved by the Institutional Ethics Committee of Anhui Medical University and was conducted according to the Declaration of Helsinki principles.

\section{Statistical analyses}

c.3321delA genotyping adhered to quality control standards, with a call rate $>95 \%$ and meeting Hardy-Weinberg equilibrium $(P>0.01)$ in the controls. The c.3321delA allelic and genotypic frequencies were compared between the $\mathrm{AD}$ patients and controls using the $\chi^{2}$ test with $2 \times 2$ and $2 \times 3$ contingency tables (SPSS 10.0, Statistical Program for Social Sciences, Illinois). The Fisher's exact test was used to compare the variable frequencies when the expected count was less than 5. Stratified analyses were performed to examine the relation between c.3321delA and the AD phenotypes. $P<0.05$ (two-tailed) was considered significant. The genetic models (dominant, recessive and additive models) were calculated for c.3321delA using logistic regression. To assess the effect of c.3321delA on the age of onset and disease severity, quantitative trait locus(QTL)analyses were performed in cases using ANOVA and nonparametric tests.

\section{Results}

\section{Characteristics of the study subjects}

The clinical characteristics of 1,080 patients (629 male and 451 female with a mean age of $5.14 \pm 6.42$ years), including age of onset, $\mathrm{AD}$ with asthma, $\mathrm{AD}$ with $\mathrm{AR}$, total $\mathrm{IgE}$ and $\mathrm{AD}$ severity, are summarized in Table 1 . The 908 controls (629 male and 451 female) have a mean age of $16.35 \pm 9.32$ years.

\section{The association of AD with c.3321delA}

The c.3321delA FLG mutation was significantly associated with AD $\quad\left(P=3.09 \quad \mathrm{E}-12, \mathrm{OR}=3.43, \quad 95 \% \mathrm{CI}=2.38-4.96 ; \quad P_{\text {genotype }}\right.$ $=1.75 \mathrm{E}-11, \mathrm{OR}=2.93,95 \% \mathrm{CI}=2.00-4.28)$. We further evaluated the homozygous and heterozygous odds ratio $\left(\mathrm{OR}_{\mathrm{hom}} / \mathrm{OR}_{\mathrm{het}}\right)$ for c.3321delA in the cases and controls. Using allele $\mathrm{A}$ as the reference allele, the $\mathrm{OR}_{\text {het }}$ estimate of c.3321delA was $2.93(95 \% \mathrm{CI}=2.00$ 4.28); however, the $\mathrm{OR}_{\text {hom }}$ estimates could not be calculated because no controls were homozygous for c.3321delA (Table 2). Overall, the genetic model analysis revealed that the additive model best described the association of c.3321delA with $\mathrm{AD}(P=3.09 \mathrm{E}-11$, $\mathrm{OR}=3.43,95 \% \mathrm{CI}=2.38-4.96)$.

\section{Clinical phenotype stratification analyses}

We also assessed the association between c.3321delA and AD phenotypes (Table 3) using stratified analyses in the cases. Significant associations were observed between c.332ldelA and concomitant skin xerosis $(P=1.68 \mathrm{E}-03, \mathrm{OR}=2.13,95 \% \mathrm{CI}=$ $1.32-3.46)$, IV $(P=2.17 \mathrm{E}-02, \quad \mathrm{OR}=1.63,95 \% \mathrm{CI}=1.07-2.49)$, palmar hyperlinearity $(P=3.64 \mathrm{E}-17, \mathrm{OR}=4.03,95 \% \mathrm{CI}=2.86-$ $5.70)$, keratosis pilaris $(P=1.72 \mathrm{E}-02, \mathrm{OR}=1.70,95 \% \mathrm{CI}=1.09$ 2.64), white dermatographism $(P=4.25 \mathrm{E}-03, \mathrm{OR}=1.82,95 \% \mathrm{CI}$ $=1.22-2.71)$ and food intolerance $(P=1.51 \mathrm{E}-03, \mathrm{OR}=1.76$, 95\% CI $=1.23-2.50)$ (Table 3). In the QTL analysis, we found that c.3321 delA was associated with disease severity $(P=9.67 \mathrm{E}-$ 05). The c.3321delA homozygous and heterozygous patients displayed a significantly increased average SCORAD score (32.87 and 30.79, respectively) compared with the patients with a wild-type genotype (25.73) (Table 4). The patients harboring c.3321delA (homozygous and heterozygous) displayed a trend of earlier age of onset ( 0.16 and 0.81 years, respectively) compared with the wild-type genotype (1.07 years), which although displayed no statistical significance but showed a trend among three groups $(P=0.056)$ (Table 4). We observed that the c.3321delA allele frequencies in $\mathrm{AD}$ patients without asthma or $\mathrm{AR}$ were slightly higher than in patients with asthma or AR, but the differences were not statistically significant (all $P>0.05$ ) (Table 3). In stratified analyses, c.332ldelA was not associated with other phenotypes of $\mathrm{AD}$, including early age of onset, elevated total serum IgE levels and orbital darkening ( $P>0.05)$ (Table 3).

\section{The relationship between age of onset and c.3321delA- associated phenotypes of $A D$}

In order to explore whether patients with earlier onset tend to present the phenotypes associated with c.3321delA or whether patients with mutation related phenotypes display a trend of earlier onset, we divided the patients into two groups (early age of onset and late age of onset), calculated these phenotypes' prevalence of the two groups, and compared the phenotypedistribution difference using the $\chi^{2}$ test. We observed that the prevalence of $\mathrm{AD}$ concomitant with $\mathrm{IV}$ in the early age of onset group was significant lower than late age of onset group $(13.37 \%$ vs $27.27 \%, P=0.004)$. On the other hand, $\mathrm{AD}$ concomitant with IV displayed a trend of later onset than without IV (1.56 years vs 0.91 years, $P=0.026)$, as well as $\mathrm{AD}$ concomitant with keratosis 
Table 1. The clinical characteristics of 1,080 cases.

\begin{tabular}{|c|c|}
\hline Phenotype & Patients \\
\hline Male (\%) & $629(58.24 \%)$ \\
\hline Female (\%) & $451(41.76 \%)$ \\
\hline Age (years), mean $\pm S D$ (range) & $5.14 \pm 6.42(0.5-58)$ \\
\hline Age of onset (years), mean $\pm S D$ (range) & $1.03 \pm 3.00(0.02-37)$ \\
\hline Early age of onset ( $\leq 2$ years) $(\%)(n *)$ & $999(92.59 \%)(1,079)$ \\
\hline AD with asthma (\%) $\left(n^{*}\right)$ & $246(22.82 \%)(1,078)$ \\
\hline AD with allergic rhinitis $(\%)\left(n^{*}\right)$ & $344(32.12 \%)(1,071)$ \\
\hline AD with xerosis $(\%)\left(n^{*}\right)$ & $812(75.19 \%)(1,080)$ \\
\hline AD with IV (\%) $\left(n^{*}\right)$ & $155(14.35 \%)(1,080)$ \\
\hline AD with palmar hyperlinearity $(\%)\left(n^{*}\right)$ & $237(22.11 \%)(1,072)$ \\
\hline AD with keratosis pilaris (\%) $\left(n^{*}\right)$ & $134(12.51 \%)(1,071)$ \\
\hline AD with orbital darkening (\%) $\left(n^{*}\right)$ & $89(8.27 \%)(1,076)$ \\
\hline AD with food intolerance $(\%)\left(n^{*}\right)$ & $407(42.66 \%)(954)$ \\
\hline AD with white dermatographism (\%) $\left(n^{*}\right)$ & $158(14.65 \%)(1,080)$ \\
\hline Elevated total lgE (>100 IU/ml) (\%) $\left(\mathrm{n}^{*}\right)$ & $575(66.45 \%)(816)$ \\
\hline Mild AD (objective SCORAD $\leq 15)(\%)\left(n^{*}\right)$ & $179(16.57 \%)(1,080)$ \\
\hline Moderate AD $(15<$ objective SCORAD $\leq 40)(\%)\left(n^{*}\right)$ & $764(70.74 \%)(1,080)$ \\
\hline Severe AD (objective SCORAD>40) (\%) $\left(n^{*}\right)$ & $137(12.69 \%)(1,080)$ \\
\hline
\end{tabular}

pilaris also had a trend of later onset than without keratosis pilaris (1.69 years vs 0.91 years, $P=0.034$ ) (Table 5). There was no significant difference between age of onset and phenotypes (xerosis, palmar hyperlinearity, food intolerance and white dermatographism) (Table 5).

\section{Discussion}

In our previous GWAS, we confirmed that the FLG mutation c.3321delA is associated with AD in the Chinese Han population [9]. In the current study, our genotype-phenotype analyses of AD may aid in the investigation of various disease phenotypes and the identification of phenotype-specific genetic factors, thereby providing new insights into the pathogenesis of $\mathrm{AD}$. Our findings indicate that c.3321delA significantly associates with various $\mathrm{AD}$ clinical phenotypes, including skin xerosis, IV, palmar hyperli- nearity, keratosis pilaris, white dermatographism, food intolerance and disease severity.

The association of c.3321delA in our group was best described with an additive model that displayed a clear trend for increased disease risk in heterozygous and homozygous c.3321delA patients. Our analysis comparing AD severity (measured by the objective SCORAD score) between the genotype groups in the QTL analysis showed that homozygous and heterozygous c.3321delA patients were more likely to have a more severe form of the disease, and this finding is consistent with the Singaporean study that showed that the combined null FLG genotype of 17 mutations detected in cases and controls were strongly associated with increased AD severity (permutation test $P=0.0063$ ) [6]. However, the association was inconsistent in other studies $[2,10,11]$ in the Chinese Han population, all of which were assessing compound genotypes of $F L G$ including c.3321delA with smaller samples. FLG

Table 2. Genotype of c. $3321 \mathrm{del} A$ in 1,080 cases and 908 control.

\begin{tabular}{|c|c|c|c|c|}
\hline & Cases $(n=1080)$ & Controls $(n=908)$ & OR $(95 \% \mathrm{Cl})$ & $P$ \\
\hline \multicolumn{5}{|l|}{ Genotype } \\
\hline AA & $949(87.87 \%)$ & $871(95.93 \%)$ & Reference & \\
\hline $\mathrm{Aa}$ & $118(10.93 \%)$ & $37(4.07 \%)$ & $2.93(2.00-4.28)$ & $1.75 \mathrm{E}-11$ \\
\hline aa & $13(1.20 \%)$ & $0(0 \%)$ & NA & \\
\hline \multicolumn{5}{|c|}{ Recessive model } \\
\hline$a a /(A a+A A)$ & $13 / 1067$ & $0 / 908$ & NA & $9.10 \mathrm{E}-04$ \\
\hline \multicolumn{5}{|c|}{ Dominant model } \\
\hline$(a a+A a) / A A$ & $131 / 949$ & $37 / 871$ & $3.25(2.23-4.73)$ & $1.26 \mathrm{E}-10$ \\
\hline \multicolumn{5}{|c|}{ Additive model } \\
\hline $\mathrm{aa} / \mathrm{Aa} / \mathrm{AA}$ & 13/118/949 & 0/37/871 & $3.43(2.38-4.96)$ & $3.09 \mathrm{E}-11$ \\
\hline
\end{tabular}


Table 3. The association between c.3321 delA and clinical phenotypes in AD.

\begin{tabular}{|c|c|c|c|c|c|}
\hline \multirow[t]{2}{*}{ Clinical phenotypes } & \multicolumn{2}{|c|}{ Allele frequencies } & \multirow[t]{2}{*}{$P$} & \multirow[t]{2}{*}{ OR } & \multirow[t]{2}{*}{$95 \% \mathrm{Cl}$} \\
\hline & 3321 delA & A & & & \\
\hline Early age of onset ( $\leq 2$ years) & 0.0691 & 0.9309 & $1.24 \mathrm{E}-01$ & 1.90 & $0.83-4.38$ \\
\hline Late age of onset ( $>2$ years) & 0.0375 & 0.9625 & & & \\
\hline AD with asthma & 0.0650 & 0.9350 & $8.60 \mathrm{E}-01$ & 0.96 & $0.64-1.45$ \\
\hline AD without asthma & 0.0673 & 0.9327 & & & \\
\hline$A D$ with $A R$ & 0.0581 & 0.9419 & $2.97 \mathrm{E}-01$ & 0.82 & $0.56-1.19$ \\
\hline$A D$ without $A R$ & 0.0702 & 0.9299 & & & \\
\hline$A D$ with elevated IgE & 0.0740 & 0.9260 & $6.59 \mathrm{E}-01$ & 1.10 & $0.77-1.56$ \\
\hline$A D$ with normal IgE & 0.0685 & 0.9315 & & & \\
\hline AD with Xerosis & 0.0764 & 0.9237 & $1.68 \mathrm{E}-03$ & 2.13 & $1.32-3.46$ \\
\hline AD without Xerosis & 0.0373 & 0.9627 & & & \\
\hline AD with IV & 0.0968 & 0.9032 & $2.17 \mathrm{E}-02$ & 1.63 & $1.07-2.49$ \\
\hline AD without IV & 0.0616 & 0.9384 & & & \\
\hline AD with Palmar hyperlinearity & 0.1519 & 0.8481 & $3.64 \mathrm{E}-17$ & 4.03 & $2.86-5.70$ \\
\hline AD without Palmar hyperlinearity & 0.0425 & 0.9575 & & & \\
\hline AD with Keratosis pilaris & 0.1007 & 0.8993 & $1.72 \mathrm{E}-02$ & 1.70 & $1.09-2.64$ \\
\hline AD without Keratosis pilaris & 0.0619 & 0.9381 & & & \\
\hline AD with Orbital darkening & 0.0506 & 0.9494 & $3.62 \mathrm{E}-01$ & 0.73 & $0.36-1.45$ \\
\hline$A D$ without Orbital darkening & 0.0684 & 0.9316 & & & \\
\hline$A D$ with food intolerance & 0.0842 & 0.9158 & $1.51 \mathrm{E}-03$ & 1.76 & $1.23-2.50$ \\
\hline$A D$ with food tolerance & 0.0496 & 0.9504 & & & \\
\hline AD with White dermatographism & 0.1107 & 0.8893 & $4.25 \mathrm{E}-03$ & 1.82 & $1.22-2.71$ \\
\hline$A D$ without White dermatographism & 0.0642 & 0.9358 & & & \\
\hline
\end{tabular}

doi:10.1371/journal.pone.0098235.t003

mutations predict dose-dependent alterations in epidermal permeability barrier function [12], and our results confirmed that the $F L G$ null mutations might serve as an indicator of severe disease phenotypes.

Several studies indicate that FLG mutations have an effect on the age of onset of $\mathrm{AD}$ such that individuals carrying FLG mutations (R501X, 2282del4, R2447X or S3247x) can lead to early-onset (age of onset $\leq 2$ years) $\mathrm{AD}$ that persisits well into adulthood [13-16]. Moreover, Ma et al. reported that c.3321delA was associated with early-onset of $\mathrm{AD}$ in Northern Chinese patients $(P=0.020)$ [17]. However, in our study, no statistical significance was observed for the association between early-onset at $\mathrm{AD}$ and $F L G$ mutation c.3321delA in stratified analysis $(P=1.24 \mathrm{E}-01)$, which may be attributed to the fact that the majority of $\mathrm{AD}$ cases begin early in life (age of onset $\leq 2$ years). It's reported that FLG mutations were associated with much earlier age at onset for AD [11,18], AD patients carrying FLG mutations were younger than those without $F L G$ mutations. However, one study in China did not observe the association $(P=0.307)$ [2]. In our study, we observed that the average/median age of onset in $\mathrm{AD}$ tended to decrease among the three groups (wide-type, homozygous and heterozygous genotype) in the QTL analysis, but there were no statistical differences $(P=0.056)$, which may be due to the low proportion of $\mathrm{AD}$ cases with homozygous c.3321delA. Further analysis using larger sample sizes will be helpful for determining the effect of c.3321delA on age of onset.

Our data provide evidence for the association between c.3321delA and various AD phenotypes, including concomitant IV, palmar hyperlinearity, and keratosis pilaris (Table 3), consistent with previous studies [6,10] regarding FLG compound mutations. A recent study in Northern China indicated that combined $F L G$ variants were significantly associated with IV and palmar hyperlinearity; however, no association with keratosis pilaris was observed [2]. These results are attributed to the fact that AD has a well-recognized association with IV [19,20] and that $F L G$ is the pathogenic gene of IV; thus, AD patients from non-IV

Table 4. Association between genotype of c.3321delA and SCORAD and age of onset in AD.

\begin{tabular}{lllll}
\hline & & & & \\
\hline Genotypes & AA & Aa & aa & \\
\hline Patients (n) & 927 & 118 & 13 & \\
Objective SCORAD (median) & $25.73(24.00)$ & $30.79(29.30)$ & $32.87(32.00)$ & $9.67 \mathrm{E}-05$ \\
Age of onset (years) (median) & $1.07(0.17)$ & $0.81(0.17)$ & $0.16(0.083)$ & 0.056 \\
\hline doi:10.1371/journal.pone.0098235.t004 & & & &
\end{tabular}




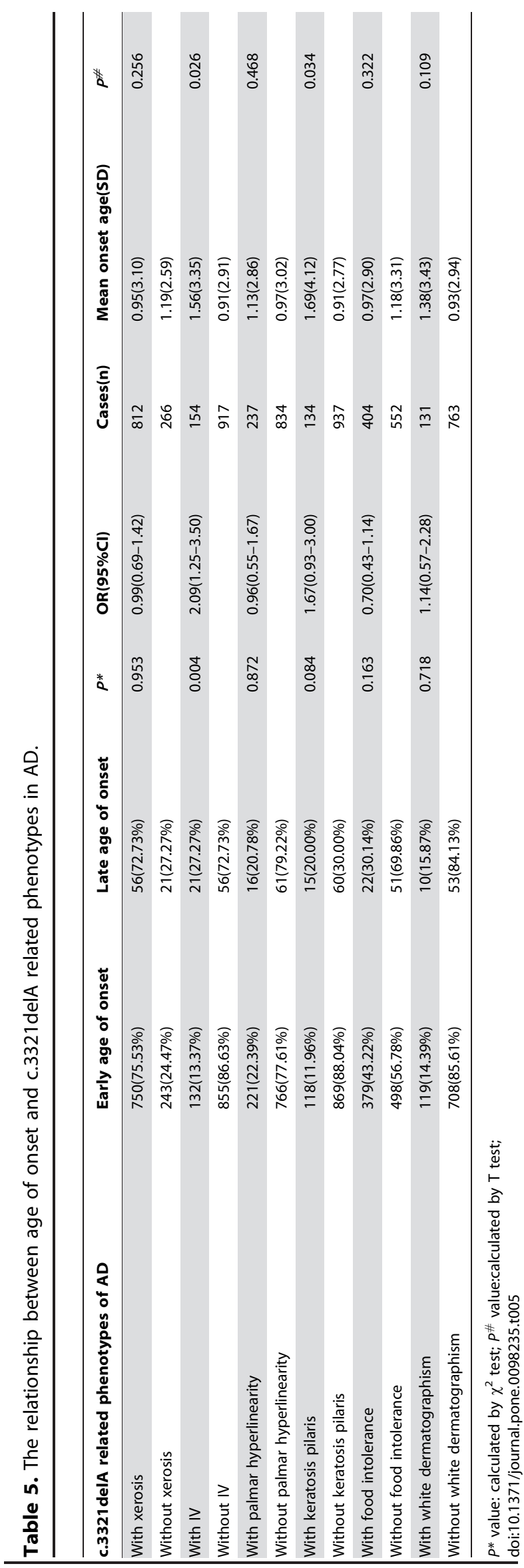

family trios have a low probability of carrying FLG mutations [10]. Hyperlinear palms and keratosis pilaris, the phenotypic characteristics of IV, have been previously reported to be strong clinical markers of $F L G$-null mutations [21]. Greater than $60 \%$ of patients carrying FLG mutations develop palmar hyperlinearity manifested as criss-cross hyperlinearity of the thenar eminence [22]. Hyperlinear palms,keratosis pilaris and IV were all skin barrer dysfunction disorders, and they have common pathogenesis with $\mathrm{AD}$, thus they could represent good phenotypic indicators of FLGnull mutations and AD.

In this study, we first reported that c.3321delA was associated with $\mathrm{AD}$ coexistent skin xerosis in Asian populations. A similar study using a smaller sample in Northern China did not find this association [2]. But the German study has reported a strong association between combined FLG mutations and dry skin [21]. $F L G$ gene number polymorphisms has been associated with the dry skin phenotype [23]. The loss or reduction of filaggrin expression disrupts barrier formation making filaggrin-deficient skin susceptible to increased transepidermal water loss and easy penetrated by environmental allergens, which can manifest as varying degrees of dry skin. The filaggrin degradation products, namely several amino acids (alanine, pyrrolidone carboxylic acid, and urocanoic acid) act as natural moisturizing factors (NMFs) in the stratum corneum. The hygroscopic NMFs are important for the maintenance of epidermal barrier hydration. NMFs are less abundant in dry skin [24], which becomes more pronounced with age [25] and changing seasons. Biochemical and immunological evidence indicate that profilaggrin and processed filaggrin are completely absent in patients carrying FLG mutations [26]. FLG deficiency or absence results in reduced NMFs and impaired epidermal barrier function, which likely contribute to the etiopathogenesis of $\mathrm{AD}$ and $\mathrm{AD}$-associated skin xerosis. Therefore, our results are the first to suggest that FLG mutations may be associated with an individual's predisposition to skin xerosis in the context of $\mathrm{AD}$.

We also were the first to report that c.3321delA was associated with $\mathrm{AD}$ coexistent white dermatographism using a large sample study of an Asian population, whereas a similar study using a smaller sample from Northern China did not observe the association [2]. White dermatographism expresses as local erythema followed by edema and a surrounding flare reaction caused by a stroke with a dull object. Dermatographism is likely considered to be caused by mechanicoimmunological stimulation of mast cells that release histamine. Mechanical trauma is thought to release an antigen that interacts with IgE-sensitized mast cells, which further release inflammatory mediators like histamine into the tissues. Filaggrin degradation can release histidine acting as putative ultraviolet photoprotector [27]. Our result indicated that FLG mutation c.3321delA might involve in the development of white dermatographism.

In addition, we found that c.3321delA was associated with food intolerance, consistent with the Southern China study [11] assessing compound genotypes for combined $F L G$ variants and the study by Linneberg et al [28] assessing FLG mutations R501X or 2282del4. FLG mutations predispose individuals to AD with allergic sensitization [29]. Studies have indicated that the absorption of allergens through the skin of patients with FLG mutations may be a predisposing factor for the development of other allergic disorders [30]. The destruction of normal epidermal barrier function is considered a key event in allergic sensitization [31]. A damaged epidermal barrier allows allergens to penetrate into the skin easily and exposes the allergens to antigen-presenting cells, causing allergic sensitization. Except skin keratinizing epithelial tissue, filaggrin is also expressed in other keratinizing 
epithelia, such as the oral and nasal mucosa, and is also presumed to contribute to the oral epithelial barrier function. Since, allergen through oral can also cause allergy. Our results confirm that skin barrier defects due to FLG mutations play a crucial role in the pathogenesis of other allergic disorders, such as food sensitization.

The relationship among $\mathrm{AD}$, asthma, $\mathrm{AR}$ and $F L G$ mutations is complex. Common and varying genetic factors for $\mathrm{AD}$ and asthma have been reported in the Chinese Han population [32]. A large European cohort study demonstrated that FLG null mutations predispose to allergic phenotypes, such as asthma and AR involved in the atopic march only in the presence of eczema [33]. Several studies have shown that FLG mutations predispose to asthma but only in the context of prior eczema or $\mathrm{AD}$ and their families, which indicating that FLG mutations did not have an independent effect on asthma [34-36]. A study [17] in Northern China reported that c.3321delA was associated with $\mathrm{AD}$-associated $\mathrm{AR}$ or asthma in stratified analysis $(P=0.035)$. However, a Polish study also found evidence for the association between combined FLG variants with asthma and $\mathrm{AR}$, and an association between $F L G$ null variants and atopic asthma was also observed in individuals without $\mathrm{AD}$ or a history thereof [37]. In one study in Northern China, no association was observed between the combined FLG mutations and $\mathrm{AD}$-associated asthma or AR; however, an association between the mutation $\mathrm{K} 4671 \mathrm{X}$ and $\mathrm{AD}$-associated $\mathrm{AR}$ was found [2]. An additional study in Southern China did not find an association between the combined FLG mutations and ADassociated asthma or AR. However, c.3321delA was found more frequently in $\mathrm{AD}$ patients without asthma than patients with asthma. In addition, a significant association between c.3321delA and $\mathrm{AD}$ patients with asthma was observed $(P=0.016)$ [11], implying that c.3321delA may serve as a protective factor for asthma that occurs in the context of AD. In this larger sample study, we observed that the c.3321delA allele frequencies in AD patients without asthma or AR were slightly increased compared with $\mathrm{AD}$ patients with asthma or AR, but the findings were not statistically significant $(P=0.86, \mathrm{OR}=0.96,95 \% \mathrm{CI}=0.64-1.45$ and $P=0.297, \quad \mathrm{OR}=0.82, \quad 95 \% \mathrm{CI}=0.56-1.19, \quad$ respectively) (Table 3). The negative association between FLG mutations and atopies found in our study may be attributed to sample bias, environmental factors and ethnic differences. The majority of our patients displayed an age of onset for asthma and AR well below the median age at onset. However, we confirmed the previous hypothesis that c.3321delA might be a protective factor for asthma. Additional studies are needed to clarify the relationship between FLG mutations and $\mathrm{AD}$-associated respiratory allergic disorders.

We also performed an analysis regarding orbital darkening, but no significant difference was found, consistent with the Northern

\section{References}

1. Barnes KC (2010) An update on the genetics of atopic dermatitis: scratching the surface in 2009. J Allergy Clin Immunol 125: 16-29 e11-11; quiz 30-11.

2. Li M, Liu Q, Liu J, Cheng R, Zhang H, et al. (2013) Mutations analysis in filaggrin gene in northern China patients with atopic dermatitis. J Eur Acad Dermatol Venereol 27: 169-174.

3. Irvine AD, McLean WH, Leung DY (2011) Filaggrin mutations associated with skin and allergic diseases. N Engl J Med 365: 1315-1327.

4. Williams H, Stewart A, von Mutius E, Cookson W, Anderson HR (2008) Is eczema really on the increase worldwide? J Allergy Clin Immunol 121: 947-954 e915.

5. Spergel JM, Paller AS (2003) Atopic dermatitis and the atopic march. J Allergy Clin Immunol 112: S118-127.

6. Chen H, Common JE, Haines RL, Balakrishnan A, Brown SJ, et al. (2011) Wide spectrum of filaggrin-null mutations in atopic dermatitis highlights differences between Singaporean Chinese and European populations. Br J Dermatol 165: $106-114$.

7. Hanifin JM RG (1980) Diagnostic features of atopic dermatitis. Acta Derm 92(Suppl.): 44-47.
China study [2]. AD patients tend to have increased IgE levels. A study from Japan reported that c.3321delA is associated with elevated IgE levels [38], but we were unable to replicate that association in this study, which is consistent with the south China [11] and Polish [37] studies.Above all, according to our results, mutation c.3321 delA was associated with several AD phenotypes, and $\mathrm{AD}$ patients with c.3321delA tended to have earlier age of onset, though the association is not significant. Since this, we performed further stratified analysis to explore the possible relationship between age of onset and these six $\mathrm{AD}$ phenotypes associated with c.3321delA. And we found that AD patients with late age of onset were more likely to accompany with IV, and AD patients with IV or keratosis pilaris tended to have later age of onset in AD. This may be due to the later predilection ages of IV and keratosis pilaris than that of $\mathrm{AD}$.

Of course, there are some limitations in our study. Our study is limited by all cases and controls being of Chinese Han and it is possible that the $F L G$ mutations may not exert the same effect in other races of China and other Asian countries. Our sample size was not large enough, so that most $(92.59 \%)$ of our patients were early age of onset ( $\leq 2$ years), and only 13 patients were homozygous, which may lead to minor bias. In addition, the phenotypes-genotypes association analysis in current study was just focused on $\mathrm{AD}$ patients, and all the results about phenotypesgenotypes relationship were in the presence of $\mathrm{AD}$. In future study, we will perform the associated analyses of c.3321delA in the non$\mathrm{AD}$ group, such as groups of $\mathrm{IV}$, asthma and $\mathrm{AR}$, et al.

In conclusion, our study confirmed that the FLG mutation c.3321delA was associated with $\mathrm{AD}$ under an additive genetic model in a large Chinese cohort. In addition, we observed a correlation between c.3321delA and various clinical features of $\mathrm{AD}$, and we demonstrated that c.3321delA has an effect on these phenotypes in the context of $\mathrm{AD}$. These findings may help to further define the role of $F L G$ in $\mathrm{AD}$ susceptibility, thereby assisting in the categorization of various subtypes of the disease and building the foundation for genetic diagnosis and personalized treatment for patients with $\mathrm{AD}$ in the near future.

\section{Acknowledgments}

We thank all study participants and all the volunteers who have so willingly participated in this study, thus make this study possible.

\section{Author Contributions}

Conceived and designed the experiments: LS X. Zhang SY. Performed the experiments: LM LW XJ JZ JG BL XF YC WY WZ BW DD CS HC. Analyzed the data: X. Zuo HT. Wrote the paper: LM. Rectified the manuscript: LM HT LS XT.

8. (1993) Severity scoring of atopic dermatitis: the SCORAD index. Consensus Report of the European Task Force on Atopic Dermatitis. Dermatology 186: 23-31.

9. Sun LD, Xiao FL, Li Y, Zhou WM, Tang HY, et al. (2011) Genome-wide association study identifies two new susceptibility loci for atopic dermatitis in the Chinese Han population. Nat Genet 43: 690-694.

10. Cheng R, Li M, Zhang H, Guo Y, Chen X, et al. (2012) Common FLG mutation K4671X not associated with atopic dermatitis in Han Chinese in a family association study. PLoS One 7: e49158.

11. Zhang H, Guo Y, Wang W, Shi M, Chen X, et al. (2011) Mutations in the filaggrin gene in Han Chinese patients with atopic dermatitis. Allergy 66: 420427.

12. Gruber R, Elias PM, Crumrine D, Lin TK, Brandner JM, et al. (2011) Filaggrin genotype in ichthyosis vulgaris predicts abnormalities in epidermal structure and function. Am J Pathol 178: 2252-2263.

13. Weidinger S, Rodriguez E, Stahl C, Wagenpfeil S, Klopp N, et al. (2007) Filaggrin mutations strongly predispose to early-onset and extrinsic atopic dermatitis. J Invest Dermatol 127: 724-726. 
14. Barker JN, Palmer CN, Zhao Y, Liao H, Hull PR, et al. (2007) Null mutations in the filaggrin gene (FLG) determine major susceptibility to early-onset atopic dermatitis that persists into adulthood. J Invest Dermatol 127: 564-567.

15. Greisenegger E, Novak N, Maintz L, Bieber T, Zimprich F, et al. (2010) Analysis of four prevalent filaggrin mutations (R501X, 2282del4, R2447X and S3247X) in Austrian and German patients with atopic dermatitis. J Eur Acad Dermatol Venereol 24: 607-610.

16. Brown SJ, Sandilands A, Zhao Y, Liao H, Relton CL, et al. (2008) Prevalent and low-frequency null mutations in the filaggrin gene are associated with early-onset and persistent atopic eczema. J Invest Dermatol 128: 1591-1594.

17. Ma L, Zhang L, Di ZH, Zhao LP, Lu YN, et al. (2010) Association analysis of filaggrin gene mutations and atopic dermatitis in Northern China. Br J Dermatol 162: $225-227$

18. Carson CG, Rasmussen MA, Thyssen JP, Menne T, Bisgaard H (2012) Clinical presentation of atopic dermatitis by filaggrin gene mutation status during the first 7 years of life in a prospective cohort study. PLoS One 7: e48678.

19. Mevorah B, Marazzi A, Frenk E (1985) The prevalence of accentuated palmoplantar markings and keratosis pilaris in atopic dermatitis, autosomal dominant ichthyosis and control dermatological patients. Br J Dermatol 112: 679-685.

20. Sandilands A, O'Regan GM, Liao H, Zhao Y, Terron-Kwiatkowski A, et al. (2006) Prevalent and rare mutations in the gene encoding filaggrin cause ichthyosis vulgaris and predispose individuals to atopic dermatitis. J Invest Dermatol 126: 1770-1775.

21. Novak N, Baurecht H, Schafer T, Rodriguez E, Wagenpfeil S, et al. (2008) Lossof-function mutations in the filaggrin gene and allergic contact sensitization to nickel. J Invest Dermatol 128: 1430-1435.

22. Brown SJ, Relton CL, Liao H, Zhao Y, Sandilands A, et al. (2009) Filaggrin haploinsufficiency is highly penetrant and is associated with increased severity of eczema: further delineation of the skin phenotype in a prospective epidemiological study of 792 school children. Br J Dermatol 161: 884-889.

23. Ginger RS, Blachford S, Rowland J, Rowson M, Harding CR (2005) Filaggrin repeat number polymorphism is associated with a dry skin phenotype. Arch Dermatol Res 297: 235-241.

24. Horii I, Nakayama Y, Obata M, Tagami H (1989) Stratum corneum hydration and amino acid content in xerotic skin. Br J Dermatol 121: 587-592.

25. Jacobson TM, Yuksel KU, Geesin JC, Gordon JS, Lane AT, et al. (1990) Effects of aging and xerosis on the amino acid composition of human skin. J Invest Dermatol 95: 296-300.

26. Smith FJ, Irvine AD, Terron-Kwiatkowski A, Sandilands A, Campbell LE, et al. (2006) Loss-of-function mutations in the gene encoding filaggrin cause ichthyosis vulgaris. Nat Genet 38: 337-342.
27. McLoone P, Simics E, Barton A, Norval M, Gibbs NK (2005) An action spectrum for the production of cis-urocanic acid in human skin in vivo. J Invest Dermatol 124: 1071-1074.

28. Linneberg A, Fenger RV, Husemoen LL, Thuesen BH, Skaaby T, et al. (2013) Association between loss-of-function mutations in the filaggrin gene and selfreported food allergy and alcohol sensitivity. Int Arch Allergy Immunol 161: $234-242$.

29. Weidinger S, Illig T, Baurecht H, Irvine AD, Rodriguez E, et al. (2006) Loss-offunction variations within the filaggrin gene predispose for atopic dermatitis with allergic sensitizations. J Allergy Clin Immunol 118: 214-219.

30. Scharschmidt TC, Man MQ Hatano Y, Crumrine D, Gunathilake R, et al. (2009) Filaggrin deficiency confers a paracellular barrier abnormality that reduces inflammatory thresholds to irritants and haptens. J Allergy Clin Immunol 124: 496-506, 506 e491-496.

31. Schuttelaar ML, Kerkhof M, Jonkman MF, Koppelman GH, Brunekreef B, et al. (2009) Filaggrin mutations in the onset of eczema, sensitization, asthma, hay fever and the interaction with cat exposure. Allergy 64: 1758-1765.

32. Tang HY, Tang XF, Zuo XB, Gao JP, Sheng YJ, et al. (2012) Association analysis of single nucleotide polymorphisms at five loci: comparison between atopic dermatitis and asthma in the Chinese Han population. PLoS One 7: e35334.

33. Marenholz I, Nickel R, Ruschendorf F, Schulz F, Esparza-Gordillo J, et al. (2006) Filaggrin loss-of-function mutations predispose to phenotypes involved in the atopic march. J Allergy Clin Immunol 118: 866-871.

34. Morar N, Cookson WO, Harper JI, Moffatt MF (2007) Filaggrin mutations in children with severe atopic dermatitis. J Invest Dermatol 127: 1667-1672.

35. Henderson J, Northstone K, Lee SP, Liao H, Zhao Y, et al. (2008) The burden of disease associated with filaggrin mutations: a population-based, longitudinal birth cohort study. J Allergy Clin Immunol 121: 872-877 e879.

36. Weidinger S, O'Sullivan M, Illig T, Baurecht H, Depner M, et al. (2008) Filaggrin mutations, atopic eczema, hay fever, and asthma in children. J Allergy Clin Immunol 121: 1203-1209 e1201.

37. Poninska J, Samolinski B, Tomaszewska A, Raciborski F, Samel-Kowalik P, et al. (2011) Filaggrin gene defects are independent risk factors for atopic asthma in a Polish population: a study in ECAP cohort. PLoS One 6: e16933.

38. Enomoto H, Hirata K, Otsuka K, Kawai T, Takahashi T, et al. (2008) Filaggrin null mutations are associated with atopic dermatitis and elevated levels of $\mathrm{IgE}$ in the Japanese population: a family and case-control study. J Hum Genet 53: 615621 . 